\title{
Alexandru Solian
}

On the extension of transi-groups

Czechoslovak Mathematical Journal, Vol. 15 (1965), No. 1, 30-36

Persistent URL: http://dml.cz/dmlcz/100650

\section{Terms of use:}

(C) Institute of Mathematics AS CR, 1965

Institute of Mathematics of the Czech Academy of Sciences provides access to digitized documents strictly for personal use. Each copy of any part of this document must contain these Terms of use.

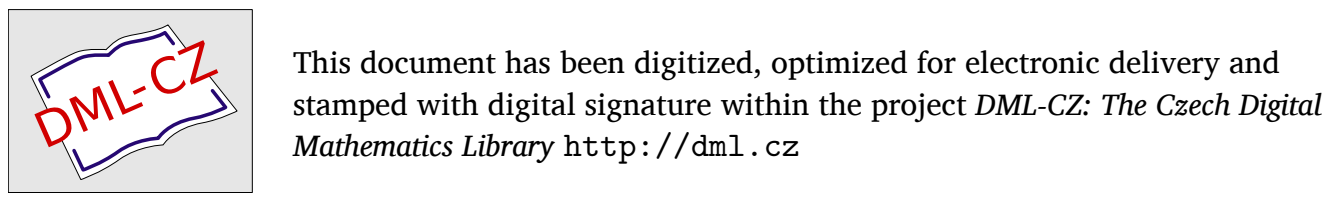




\title{
ON THE EXTENSION OF TRANSI-GROUPS
}

\author{
Alexandru Solian, (Bucharest)
}

(Received September 29, 1963)

The author generalizes the theory of extensions of groups to the transigroups introduced by the author in the paper [5].

In the theory of transi-groups one can construct the quotient-transi-group of a given transi-group with respect to a normal subtransi-group of the latter. As in the theory of groups one can raise the problem of finding transi-groups which are in a certain sense, extensions of two given transi-groups. The present paper treats and solves a slightly modificated problem.

Coming now to a more precise formulation we remark that, when giving a transigroup $(M, \mathfrak{A}, e)$ and an equivalence relation $\sim$ in $M$, imprimitive with respect to $\mathfrak{A}$, i.e. a normal subtransi-group $(K, \mathfrak{B}, e, \sim)$, the group $\mathfrak{B}$ is not an (effective) transformation group of $K$ but it is homorphically mapped onto such a group $\mathfrak{B}^{*}$, obtaining in this manner the transi-group $\left(K, \mathfrak{B}^{*}, e\right)$ associated with $(K, \mathfrak{B}, e, \sim)$. The group $\mathfrak{A}$ is an extension of $\mathfrak{B}$ by $\mathfrak{A} / \mathfrak{B}$ (which acts as a transformation group of $M / \sim$ ). If the quotient-transi-group $(M / \sim, \mathfrak{A} / \mathfrak{B},[e])$ (where $[e]$ is the equivalence class of $e$ and coincides as a subset of $M$ with $K$ ) is transitive, all the equivalence classes of $M$ with respect to $\sim$ have the same cardinal number, so that $M$ can be identified with the cartesian product $K \times(M / \sim)$.

The present paper deals with the problem of existence (and not of finding all solutions) of a transi-group $(M, \mathfrak{A}, e)$ when the group $\mathfrak{A}$ is given as an extension of $\mathfrak{B}$ by $\mathfrak{A} / \mathfrak{B}$. To be more precise, a necessary and sufficient condition is given in order that the given extension $\mathfrak{A}$ act as an (effective) transformation group in the cartesian product $K \times(M / \sim)$.

For the questions of the theory of equivalence relations, the reader may refer to [2] and [1], for those of the theory of groups to [3] and for those of the theory of transigroups, to the papers [4] and [5] where the definitions of technical terms employed are also given.

1. Let $K$ be a set and let $\mathfrak{B}$ be a group for which there exists a group homomorphism $\psi$ from $\mathfrak{B}$ onto an (effective) transformation group $\mathfrak{B}^{*}$ of $K$. Let $\left(M^{*}, \mathfrak{A}^{*}, e^{*}\right)$ 
be a transitive transi-group; let $\mathfrak{I}_{0}^{*}$ be the subgroup of all $\alpha, \alpha \in \mathfrak{A}^{*}$, for which $\alpha\left(e^{*}\right)=$ $=e^{*}$ and let $\alpha_{\lambda}, \lambda \in \Lambda$, be a system of representatives of the left residue classes of $\mathfrak{A}^{*}$ with respect to $\mathfrak{A}_{0}^{*}$ (here the representative of $\mathfrak{I}_{0}^{*}$ is meant to be $\varepsilon$, the unit element of $\left.\mathfrak{A}^{*}\right)$.

For any $x^{*} \in M^{*}$, there exists a unique $\lambda, \lambda \in \Lambda$, for which $\alpha_{\lambda}\left(e^{*}\right)=x^{*}$.

Let $\mathfrak{A}$ be a given extension of $\mathfrak{B}$ by $\mathfrak{A}^{*}$; let $\tau_{\alpha}, \alpha \in \mathfrak{A}^{*}$, be a system of representatives of the extension, where $\tau_{\varepsilon}=1$ (here 1 denotes the unit element of $\mathfrak{A}$ ); let $\mu_{\alpha, \beta}, \alpha, \beta \in \mathfrak{A}^{*}$, be the factor system associated with the $\tau_{\alpha}$. Let us denote by $\mathfrak{A}_{0}$ the subgroup of all elements of $\mathfrak{A}$, whose residue classes are in $\mathfrak{A}_{0}^{*}$; we have $\mathfrak{A}_{0} \supset \mathfrak{B}$. We introduce the notations

$$
\sigma^{(\alpha)}=\tau_{\alpha} \sigma \tau_{\alpha}^{-1}, \quad \sigma^{\alpha}=\tau_{\alpha}^{-1} \sigma \tau_{\alpha}, \quad \alpha \in \mathfrak{A}^{*}, \quad \sigma \in \mathfrak{B}
$$

The elements of $\mathfrak{A}$ may be uniquely represented in the form $\sigma \tau_{\alpha}, \sigma \in \mathfrak{B}, \alpha \in \mathfrak{A} *$ and the product is made according to

$$
\left(\sigma_{1} \tau_{\alpha}\right)\left(\sigma_{2} \tau_{\beta}\right)=\sigma_{1} \sigma_{2}^{(\alpha)} \mu_{\alpha, \beta} \tau_{\alpha \beta}, \quad \alpha, \beta \in \mathfrak{A}^{*}, \quad \sigma_{1}, \sigma_{2} \in \mathfrak{B}
$$

Let us denote the cartesian product $K \times M^{*}$ by $M$.

Finally we make the following hypothesis:

Hypothesis (S): If $\sigma, \sigma \in \mathfrak{B}$, has the property $\psi\left(\sigma^{\alpha_{\lambda}}\right)=\psi(1)$ for all $\lambda \in \Lambda$, then $\sigma=1$.

The introduction of this hypothesis will be justified at point 2 .

2. To justify the introduction of hypothesis $(\mathscr{S})$, we make the following considerations:

Suppose that $(M, \mathfrak{A}, e)$ is a transi-group and that $(K, \mathfrak{B}, e, \sim)$ is a normal subtransigroup, the quotient-transi-group with respect to whom, $\left(M^{*}, \mathfrak{I}^{*}, e^{*}\right)$ say, is transitive; it is well-known ${ }^{1}$ ) that in this case, all classes in the decomposition of $M$ by $\sim$ are cardinally equivalent; consequently, $M$ can be identified with the cartesian product $K \times M^{*}$. The identification is made in the following manner (we employ the notations of point 1 , though we have to do here with another situation):

If $x \in M$, let $x^{*}$ be his equivalence class with respect to $\sim$; suppose that $\alpha_{\lambda}, \lambda \in \Lambda$, is such that $\alpha_{\lambda}\left(e^{*}\right)=x^{*}$; then

$$
\tau_{\alpha_{\lambda}}^{-1}(x)=k \in K
$$

as is easily seen from the definition of quotient-transi-groups; we then identify $x$, $x \in M$, with the couple $\left(k, x^{*}\right) \in K \times M^{*}$.

In particular we identify $k \in K$ with $\left(k, e^{*}\right)$ and if $\alpha_{\lambda}\left(e^{*}\right)=x^{*}$ we obtain from (1), $\tau_{\alpha_{\lambda}}\left(k, e^{*}\right)=\left(k, x^{*}\right)$.

\footnotetext{
1) See, for instance, [4].
} 
With every $\sigma \in \mathfrak{B}$ there is associated, in a natural way a transformation $\psi(\sigma)$ of $K$, namely that which satisfies $\left.\left((\psi \sigma)(k), e^{*}\right)=\sigma\left(k, e^{*}\right), k \in K^{2}\right)$. Consequently, with the same notations as before, we have

$$
\sigma\left(k, x^{*}\right)=\sigma \tau_{\alpha_{\lambda}}\left(k, e^{*}\right)=\tau_{\alpha_{\lambda}}\left(\left(\psi \sigma^{\alpha_{\lambda}}\right)(k), e^{*}\right)=\left(\left(\psi \sigma^{\alpha_{\lambda}}\right)(k), x^{*}\right) .
$$

Hence, it is sen that if $\psi \sigma^{\alpha_{\lambda}}$ is the identical transformation of $K$ for all $\lambda \in \Lambda$, then $\sigma=1$.

Thus, hypothesis $(\mathscr{S})$ is a necessary condition for the construction of the transigroup $(M, \mathfrak{A}, e)$.

It must be also observed that if $\mathfrak{B}$ coincides with $\mathfrak{B}^{*}$ and $\psi$ is the identity mapping, hypothesis $(\mathscr{S})$ is automatically satisfied since among the $\sigma^{\alpha_{\lambda}}$ may be found $\sigma$ too.

3. With the data and notations of point 1 , let us suppose in addition that there exists a homomorphism $\varphi, \varphi: \mathfrak{A}_{0} \rightarrow \mathfrak{I}(K)$ whose restriction to $\mathfrak{B}$ coincides with $\psi .{ }^{3}$ )

1) We define a mapping $\eta, \eta: \mathfrak{A} \rightarrow \mathfrak{T}(M)$ in the following manner: Let $\sigma \tau_{\alpha} \in \mathfrak{A}$, $\sigma \in \mathfrak{B}, \alpha \in \mathfrak{A}^{*}$ and let $\left(k, x^{*}\right) \in M$, where $k \in K, x^{*} \in M^{*}$. If the chosen representative of the left residue class with respect to $\mathfrak{H}_{0}^{*}$, which contains $\alpha$, is $\alpha_{\varrho}$, $\varrho \in \Lambda$, then $\alpha_{\varrho}\left(e^{*}\right)=\alpha\left(e^{*}\right)$ hence $\tau_{\alpha_{\varrho}}^{-1} \tau_{\alpha} \in \mathfrak{A}_{0} ;$ put

$$
\delta_{\alpha}=\varphi\left(\tau_{\alpha_{\varrho}}^{-1} \tau_{\alpha}\right)
$$

the $\delta_{\alpha}, \alpha \in \mathfrak{2}^{*}$, define a function which associates with every $\alpha \in \mathfrak{H}^{*}$ a $\delta_{\alpha} \in \mathfrak{Z}(K)$. Suppose that $x^{*}=\alpha_{\lambda}\left(e^{*}\right)$ and that $\left.\alpha_{v} \equiv \alpha \alpha_{\lambda}\left(\bmod \mathfrak{A}_{0 s}^{*}\right),{ }^{4}\right) \lambda, v \in \Lambda$.

Then, by definition, ${ }^{5}$ )

$$
\left(\eta\left(\sigma \tau_{\alpha}\right)\right)\left(k, x^{*}\right)=\left(\left(\sigma \mu_{\alpha, \alpha_{\lambda}}\right)^{\alpha_{\lambda}} \delta_{\alpha \alpha_{\lambda}}(k), \alpha\left(x^{*}\right)\right) .
$$

We assert that $\eta\left(\sigma \tau_{\alpha}\right), \sigma \in \mathfrak{B}, \alpha \in \mathfrak{A}^{*}$, is a transformation of $M$ : indeed, if $\left(k^{\prime}, y^{*}\right) \in$ $\in M, k^{\prime} \in K, y^{*} \in M^{*}$, then, since $\mathfrak{A}^{*}$ acts transitively on $M$, there exist $\alpha, \alpha_{\lambda}, \alpha_{v} \in \mathfrak{A}^{*}$ where $\lambda, v \in \Lambda$ such that

$$
\alpha\left(x^{*}\right)=y^{*}=\alpha_{v}\left(e^{*}\right) \text { and } \alpha_{\lambda}\left(e^{*}\right)=x^{*} ;
$$

let $k, k \in K$, be such that

$$
\left(\sigma \mu_{\alpha, \alpha_{\lambda}}\right)^{\alpha_{v}} \delta_{\alpha \alpha_{\lambda}}(k)=k^{\prime}
$$

such a $k$ exists since $\delta_{\alpha \alpha_{\lambda}}$ and $\psi\left(\left(\sigma \mu_{\alpha, \alpha_{\lambda}}\right)^{\alpha_{\nu}}\right)$ are transformations of $K$; we have $\left(\eta\left(\sigma \tau_{\alpha}\right)\right)\left(k, x^{*}\right)=\left(k^{\prime}, y^{*}\right)$.

\footnotetext{
${ }^{2}$ ) Instead of $\sigma \tau_{\boldsymbol{\varepsilon}}$, we have written $\sigma$ which is the same thing.

$\left.{ }^{3}\right)$ By $\mathfrak{Z}(E)$ we denote, generally, the group of all transformations of the set $E$.

$\left.{ }^{4}\right)$ That is to say, left congruent with respect to $\mathfrak{A}_{0}^{*}$.

$\left.{ }^{5}\right)$ Here, instead of $\left(\psi\left(\sigma_{1}\right)\right)\left(k_{1}\right)$ we have written, in order to simplify notations, $\sigma_{1}\left(k_{1}\right), \sigma_{1} \in \mathfrak{B}$, $k_{1} \in K$.
} 
If $\left(\eta\left(\sigma \tau_{\alpha}\right)\right)\left(k, x^{*}\right)=\left(\eta\left(\sigma \tau_{\alpha}\right)\right)\left(k_{1}, x_{1}^{*}\right)$, it is an immediate fact that $x^{*}=x_{1}^{*}$; further it may be easily checked that $k=k_{1}$.

2) In order to prove that $\eta$ is a group homomorphism, let $\sigma_{1} \tau_{x}, \sigma_{2} \tau_{\beta} \in \mathfrak{I}$ and $\left(k, x^{*}\right) \in M$ where the meaning of notations is obvious; if

$$
x^{*}=\alpha_{\lambda}\left(e^{*}\right), \quad \alpha\left(x^{*}\right)=\alpha_{v}\left(e^{*}\right), \quad \beta \alpha\left(x^{*}\right)=\alpha_{\pi}\left(e^{*}\right),
$$

where $\lambda, v, \pi \in \Lambda$, then according to definition (2), we have

$$
\begin{gathered}
\eta\left(\sigma_{2} \tau_{\beta}\right) \eta\left(\sigma_{1} \tau_{\alpha}\right)\left(k, x^{*}\right)=\eta\left(\sigma_{2} \tau_{\beta}\right)\left(\left[\sigma_{1} \mu_{\alpha, \alpha_{\lambda}}\right]^{\alpha_{\nu}} \delta_{\alpha \alpha_{\lambda}}(k), \alpha\left(x^{*}\right)\right)= \\
=\left(\left[\sigma_{2} \mu_{\beta, \alpha_{\nu}}\right]^{\alpha_{\pi}} \delta_{\beta \alpha_{\nu}}\left[\left(\sigma_{1} \mu_{\alpha_{\alpha} \alpha_{2}}\right)^{\alpha_{\nu}} \delta_{\alpha \alpha_{\lambda}}(k)\right], \beta \alpha\left(x^{*}\right)\right)
\end{gathered}
$$

According to the same definition we have

$$
\eta\left(\sigma_{2} \tau_{\beta} \cdot \sigma_{1} \tau_{\alpha}\right)\left(k, x^{*}\right)=\left(\left[\sigma_{2} \sigma_{1}^{(\beta)} \mu_{\beta, \alpha} \mu_{\beta \alpha, \alpha_{\lambda}}\right]^{\alpha_{\pi}} \delta_{\beta \alpha \alpha_{\lambda}}(k), \beta \alpha\left(x^{*}\right)\right) .
$$

From relations (3) we obtain

$$
\delta_{\beta \alpha_{v}}=\varphi\left(\tau_{\alpha_{\pi}}^{-1} \tau_{\beta \alpha_{v}}\right), \quad \delta_{\alpha \alpha_{\lambda}}=\varphi\left(\tau_{\alpha_{v}}^{-1} \tau_{\alpha \alpha_{\lambda}}\right), \quad \delta_{\beta \alpha_{\lambda}}=\varphi\left(\tau_{\alpha_{\pi}}^{-1} \tau_{\beta \alpha \alpha_{\lambda}}\right) .
$$

Based on the fact that $\psi$ is a rectriction of $\varphi$ and on relations (6), the first component of the second member of (4) respectively (5), is the result of the action on $k$ of the transformation

$$
\begin{gathered}
\varphi\left(\tau_{\alpha_{\pi}}^{-1} \sigma_{2} \mu_{\beta, \alpha_{\nu}} \tau_{\alpha_{\pi}}\right) \varphi\left(\tau_{\alpha_{\pi}}^{-1} \tau_{\beta \alpha_{\nu}}\right) \varphi\left(\tau_{\alpha_{\nu}}^{-1} \sigma_{1} \mu_{\alpha, \alpha_{\lambda}} \tau_{\alpha_{\nu}}\right) \varphi\left(\tau_{\alpha_{\nu}}^{-1} \tau_{\alpha \alpha_{\lambda}}\right)= \\
=\varphi\left(\tau_{\alpha_{\pi}}^{-1} \sigma_{2} \tau_{\beta} \sigma_{1} \tau_{\alpha} \tau_{\alpha_{\lambda}}\right)
\end{gathered}
$$

respectively of the transformation

$$
\varphi\left(\tau_{\alpha_{\pi}}^{-1} \sigma_{2} \tau_{\beta} \sigma_{1} \tau_{\beta}^{-1} \mu_{\beta, \alpha} \mu_{\beta \alpha, \alpha_{\lambda}} \tau_{\alpha_{\pi}}\right) \varphi\left(\tau_{\alpha_{\pi}}^{-1} \tau_{\beta \alpha \alpha_{\lambda}}\right)=\varphi\left(\tau_{\alpha_{\pi}}^{-1} \sigma_{2} \tau_{\beta} \sigma_{1} \tau_{\alpha} \tau_{\alpha_{\lambda}}\right) .
$$

Here we used the definition of $\mu_{\xi, \omega}$ namely

$$
\tau_{\xi} \tau_{\omega}=\mu_{\xi, \omega} \tau_{\xi, \omega}
$$

So it was proved that $\eta$ is a group homomorphism.

3) Suppose that $\sigma \tau_{x}, \sigma \tau_{\alpha} \in \mathfrak{A}$, has the property that $\eta\left(\sigma \tau_{\alpha}\right)$ is the identical transformation of $M$; consequently

$$
\eta\left(\sigma \tau_{\alpha}\right)\left(k, x^{*}\right)=\left(k, x^{*}\right)
$$

for any $\left(k, x^{*}\right) \in M$.

According to definition (2), this implies, firstly, $\alpha=\varepsilon$. Let $x^{*}=\alpha_{\lambda}\left(e^{*}\right)$; since $\delta_{\alpha_{\curlywedge}}$ is the identical transformation of $K$, we obtain from the same definition (2) that $\psi\left(\sigma^{\alpha_{\lambda}}\right)$ is the identical transformation of $K$; since $x^{*}$ is arbitrarily chosen in $M^{*}, \lambda$ may be considered as an arbitrary element of $\Lambda$, so that we may apply hypothesis $(\mathscr{S})$ and obtain $\sigma=1$. 
So $\eta$ is not merely a homomorphism, but a monomorphism.

While identifying $\sigma \tau_{\alpha}$ with $\eta\left(\sigma \tau_{\alpha}\right)$ and, consequently, $\mathfrak{A}$ with $\eta(\mathfrak{A})$ we may say that $\mathfrak{A}$ is an (effective) transformation group of $M$. Moreover, if we choose in $K$ a welldetermined but otherwise arbitrary element $k_{0}$, we obtain the transi-group $(M, \mathfrak{A}, e)$ where $e=\left(k_{0}, e^{*}\right)$.

4) We define in $M$ the following equivalence relation which we denote by $\sim$ : let $\left(k_{1}, x^{*}\right),\left(k_{2}, y^{*}\right) \in M$; then, by definition $\left(k_{1}, x^{*}\right) \sim\left(k_{2}, y^{*}\right)$ if and only if $x^{*}=y^{*}$.

Considering the subgroup $\mathfrak{B}_{\mathfrak{A}}(\sim)$ associated with $\sim$, if $\sigma \tau_{\alpha}, \sigma \tau_{\alpha} \in \mathfrak{A}$, belongs to this subgroup, then, according to (2), $\alpha\left(x^{*}\right)=x^{*}$ for any $x^{*} \in M^{*}$ and then $\alpha=\varepsilon$ and $\sigma \tau_{\alpha} \in \mathfrak{B}$; conversely, every element of $\mathfrak{B}$ may be written in the form $\sigma \tau_{\varepsilon}$ and, consequently, belongs to $\mathfrak{B}_{\mathfrak{A}}(\sim)$; hence $\mathfrak{B}_{\mathfrak{A}}(\sim)=\mathfrak{B}$.

It is easily seen that the equivalence $\sim$ is imprimitive with respect to $\mathfrak{A}$.

On the other hand, the equivalence class of $e$ is $K \times\left\{e^{*}\right\}$; if we identify the couple $\left(k, e^{*}\right)$ with $k$, for any $k \in K$, then $K \times\left\{e^{*}\right\}$ is obtained to be identified with $K$. So we have to do with the normal subtransi-group $(K, \mathfrak{B}, e, \sim)$ of $(M, \mathfrak{A}, e)$.

Let $\sigma \tau_{\varepsilon} \in \mathfrak{B}$; then, from (2) we have

$$
\sigma \tau_{\varepsilon}\left(k, e^{*}\right)=\left((\psi \sigma)(k), e^{*}\right)
$$

so that $\sigma$, considered as an element of $\mathfrak{A}$, induces in $K$ the transformation $\psi(\sigma)$. Consequently, the transi-group associated with $(K, \mathfrak{B}, e, \sim)$ is $\left(K, \mathfrak{B}^{*}, e\right)$ where, namely, to $\sigma, \sigma \in \mathfrak{B}$, we associate $\psi(\sigma)$.

Now, we define a homomorphism of transi-groups, $(h, \chi),(h, \chi):(M, \mathfrak{A}, e) \rightarrow$ $\rightarrow\left(M^{*}, \mathfrak{I}^{*}, e^{*}\right)$, through the relations:

$$
h\left(k, x^{*}\right)=x^{*}, \quad \chi\left(\sigma \tau_{\alpha}\right)=\alpha, \quad k \in K, \quad x^{*} \in M^{*}, \quad \sigma \in \mathfrak{B}, \quad \alpha \in \mathfrak{A}^{*} .
$$

It was no error while anticipating on the homomorphism character of $(h, \chi)$, since according to definition (2), we have

$$
h\left(\sigma \tau_{\alpha}\left(k, x^{*}\right)\right)=\alpha\left(x^{*}\right)=\left(\chi\left(\sigma \tau_{\alpha}\right)\right)\left(h\left(k, x^{*}\right)\right)
$$

and there is no difficulty to prove the other properties of transi-group homomorphisms. Obviously, the kernel of $(h, \chi)$ is $(K, \mathfrak{B}, e, \sim)$.

5) To summarize, we have constructed a transi-group $(M, \mathfrak{A}, e)$ with the following properties:

i) Denoting by $\sim$ the equivalence defined as at 4 ), the subtransi-group generated by $\sim$ is $(K, \mathfrak{B}, e, \sim)$ where $K$ appears through identification with $K \times\left\{e^{*}\right\}$, the identification having been done as at 4$)$.

ii) For any $\sigma \in \mathfrak{B}, k \in K$, we have (7), hence the transi-group associated with $(K, \mathfrak{B}, e, \sim)$ is $\left(K, \mathfrak{B}^{*}, e\right)$ where to $\sigma, \sigma \in \mathfrak{B}$, we associate $\psi(\sigma)$.

iii) The couple $(h, \chi)$ as defined at 4$)$ is a transi-group homomorphism $(h, \chi)$ : $(M, \mathfrak{A}, e) \rightarrow\left(M^{*}, \mathfrak{I}^{*}, e^{*}\right)$, whose kernel is $(K, \mathfrak{B}, e, \sim)$. 
4. With the data and notations of point 1 , let us suppose that there exists a transigroup $(M, \mathfrak{A}, e)$, where $e=\left(k_{0}, e^{*}\right)$ such that conditions i)-iii) from 3.5) are fulfilled.

Let $\sigma \tau_{\alpha}=\mathfrak{A}_{0}$, where $\sigma \in \mathfrak{B}, \alpha \in \mathfrak{A}_{0}^{*}$; if $\left(k, e^{*}\right) \in K$ then, according to condition iii) from 3.5), we have

$$
h\left(\sigma \tau_{\alpha}\left(k, e^{*}\right)\right)=\alpha\left(e^{*}\right)=e^{*} .
$$

Hence $\sigma \tau_{\alpha}$ maps any element of $K$ in an element of $K$. Since $\mathfrak{A}_{0}$ is a group, it is not difficult to prove that $\sigma \tau_{\alpha}$ induces quite a transformation $\varphi\left(\sigma \tau_{\alpha}\right)$ of $K$. So, we have constructed a mapping $\varphi, \varphi: \mathfrak{A}_{0} \rightarrow \mathfrak{Z}(K)$, which is obviously a group homomorphism.

If $\sigma \tau_{\alpha} \in \mathfrak{B}$, then $\alpha \doteq \varepsilon$ and, by definition, $\varphi\left(\sigma \tau_{\alpha}\right)\left(k, e^{*}\right)=\sigma \tau_{\varepsilon}\left(k, e^{*}\right)$ (where in the second member we mean the result of the action of $\sigma \tau_{\varepsilon}$ on $\left(k, e^{*}\right)$ as in $\left.(M, \mathfrak{A}, e)\right)$. But according to condition ii) from 3.5) this implies that

$$
\varphi\left(\sigma \tau_{\alpha}\right)\left(k, e^{*}\right)=\left([\psi \sigma](k), e^{*}\right) .
$$

With the convention of identification (see i) from 3.5)) and on account of the fact that $\tau_{\varepsilon}=1$, relation (8) becomes

$$
(\varphi \sigma)(k)=(\psi \sigma)(k) ;
$$

as this is valid for any $k \in K$, it follows that $\psi$ is the restriction of $\varphi$ to $\mathfrak{B}$.

5. We are now quite ready to formulate our fundamental result (we are given the data and notations of point 1):

Theorem. In order that a transi-group $(M, \mathfrak{A}, e)$ (where $\left.e=\left(k_{0}, e^{*}\right), k_{0} \in K\right)$ should exist, so that conditions i)-iii) from 3.5) be satisfied, it is necessary and sufficient that there should exist a group homomorphism $\varphi, \varphi: \mathfrak{A}_{0} \rightarrow \mathfrak{I}(M)$, whose restriction to $\mathfrak{B}$ be $\psi$.

6. As particular cases we may consider the following quite trivial ones. Suppose in addition to the conditions stipulated at point 1 , that:

1) $\mathfrak{A}^{*}$ is a regular $\left.{ }^{6}\right)$ transformation group of $M^{*}$; in this case $\mathfrak{A}_{0}^{*}$ is the identity subgroup, hence $\mathfrak{A}_{0}=\mathfrak{B}$ and we may take $\varphi=\psi$.

2) $\mathfrak{A}$ is the direct product $\mathfrak{B} \times \mathfrak{I}^{*}$; in this case we may define $\varphi\left(\sigma \tau_{\alpha}\right)=\psi(\sigma)$, $\sigma \in \mathfrak{B}, \alpha \in \mathfrak{A}_{0}^{*}$.

\section{References}

[1] O. Borůvka: Lehrbuch der Gruppoid und Gruppentheorie, DVW, Berlin, 1960.

[2] P. Dubreil: Algèbre, II-ème edition, Gauthier-Villars, Paris, 1954.

\footnotetext{
$\left.{ }^{6}\right)$ For the definition see, for instance, [3], p. 36.
} 
[3] A. Г. Курои: Теория групп, Издание второе, переработанное, ГИТТЛ, Москва, 1953.

[4] A. Solian: Théorie des transi-groupes I. Relations d'équivalence et sous-groupes associés. Revue Roumaine de math. p. et appl., 9, 1964, 211-228.

[5] A. Solian: Théorie des transi-groupes II. Définitions fondamentales et théorèmes d'isomorphisme. Revue Roumaine de math. p. et appl., 9, 1964, 677-695.

Резюме

О РАСШИРЕНИИ ТРАНЗИГРУПП

АЛЕКСАНДРУ СОЛИАН (Alexandru Solian), Бухарест

Автор обобщает теорию расширения групп на случай транзигрупп, введенных им в работе [5]. 